\title{
Risk factors, clinical features and outcome of treatment of work related musculoskeletal disorders in on-site clinics among IT companies in India
}

\author{
Authors: Deepak Sharan ${ }^{1}$, Ajeesh PS ${ }^{1}$, Rameshkumar $\mathrm{R}^{1}$ and Jeena Jose ${ }^{1}$ \\ Address for correspondence: ${ }^{1}$ RECOUP Neuromusculoskeletal Rehabilitation Centre, Bangalore, India.
}

\begin{abstract}
Workrelated musculoskeletal disorders among the IT professional is a common area of concern worldwide. This study was taken up to analyze the prevalence of risk factors, clinical features and outcome of treatment in onsite clinics in vaious information technology companies in India. Result revealed poor office ergonomics (54\%), lack of keyboard tray (25\%), lack of mouse tray $(35 \%)$, lack of foot rest $(60 \%)$, improper monitor height $(80 \%)$ were the major self reported risk factors. Major identified MSD were Myofascial Pain Syndrome (49.20\%), Thoracic outlet syndrome (25.02\%), Fibromyalgia syndrome $(8.5 \%)$. Majorly affected body regions were neck (64.9\%), shoulder $(42.1 \%)$, lower back (56.5\%) and thigh $(34.2 \%)$. The results were comparable with the literature. Feedback of the participants also revealed most of the participants were well satisfied with SHARAN's protocol.
\end{abstract}

Key words: "Work related musculoskeletal disorders" "SHARAN'S® Protocol" "On-site employee health clinics"

\section{Introduction}

Work related musculoskeletal disorders (WRMSD's) are a constellation of disorders common in (but not exclusive to) computer users which involves muscle, fascia, tendon and/or neurovascular structures prevalent in neck, back and upper limb along with other parts of the body. In the recent years, WRMSDs among computer users has intensified all over the world. In a study done in USA in 1995 the prevalence of musculoskeletal symptoms has been reported as high as $61 \%$ in the neck and shoulder and $30 \%$ in the arm and hand [4]. The etiology of WRMSD is complex and controversial physical environmental factors, organizational factors, and individual factors likely play a role. Chronic exposure to these risk factors may produce cumulative trauma to musculotendinous tissue, and if there is insufficient time or capacity for the tissue to heal, WRMSD may result. Several studies carried out on computer workers in India and it revealed a high prevalence of mus- culoskeletal discomfort among Information Technology (IT) workers [1, 2, 5, 6]. Long working hours, Static postures, poor office ergonomics, and repetitive nature of work were identified as some of the risk factors leading to pain and discomfort $[2,6]$. Studies have also shown that the IT professionals were exposed to such different risk factors and therefore, it is expected that they are prone to develop workrelated musculoskeletal discomfort [7]. Keeping that in mind a study was formed to analyze the prevalence of risk factors, clinical features and outcome of treatment in onsite clinics in various information technology companies in India.

\section{Methodology}

The data presented in this study were extracted from IT professionals those who took appointment for the on-site clinic at various companies across Bangalore, Delhi and Hyderabad, India. The objective of the study was explained to all the participants

Corresponding author, Email ID: deepak.sharan@recoup.in

${ }^{1}$ Address: \#312, 80 Feet Road, 10th Block, Further Extension of Anjanapura Layout, Bangalore-560062, India 
who participated in this study and signed informed consents were obtained from the employees. The data was derived from an interval of five years (2005 2010). Demographic data were collected. Workstation risk factors were collected through a structured questionnaire. Employee's feedbacks were also used for evaluating the status of musculoskeletal health of the IT professional. Physician's diagnosis revealed type and severity of clinical features. The workstation evaluation was done by measuring chair height, table height, keyboard height and monitor height. The outcome measures used are Visual Analogue Scale for Subjective Pain, Physicians diagnosis for clinical features, Pain body diagram chart, ergonomic workstation evaluation report and patient feedback questionnaire.

Based on the severity of symptoms diagnosed by the physical therapist, the Participants received a treatment using a sequenced protocol. The treatment protocol developed by RECOUP Neuromusculoskeletal Rehabilitation Centre, Bangalore in 2005 called the "SHARAN'S ${ }^{\circledR}$ Protocol". This is an acronym of Skilled Hands-on Approach for Release of myofascia, Articular, Neural and Soft-tissue mobilisation. There are four phases in the protocol for treatment of severe discomfort, moderate discomfort, mild discomfort and maintenance phase as illustrated in Figure 1.

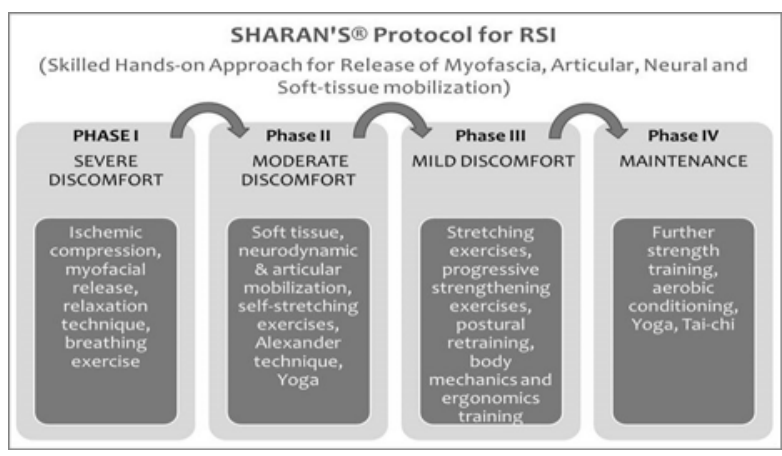

Figure 1 Overview of SHARAN'S ${ }^{\circ}$ Protocol for treatment of WRMSD

\section{Results}

The retrospective study covered 3053 IT Professionals. Statistical analyses were conducted with the Statistical Package for Social Sciences (SPSS) version 18. Based on the statistical analysis we found that primarily pain is the major feature $(95 \%)$ of dis- comfort. The mean age of the participants was $29 \pm$ 4.5 years.

The distribution of laptop and desktop users were almost equal (OR: 1.21, CI: from 0.70 to 2.1 ). $69 \%$ of the Populations were working for 5-9 hours and 30\% of the populations were working 10-14 hours.

The major self reported workstation risk factors were poor office ergonomics, lack of keyboard tray, lack of mouse tray, lack of foot rest, improper monitor height. The percentage was presented in Table 1.

Table 1.

Percentage of self reported workstation risk factors Ergonomic risk Percentage (\%) factors

\begin{tabular}{ll}
\hline $\begin{array}{l}\text { poor office ergo- } \\
\text { nomics }\end{array}$ & $54 \%$ \\
\hline $\begin{array}{l}\text { lack of keyboard } \\
\text { tray }\end{array}$ & $25 \%$ \\
\hline
\end{tabular}

tray

\begin{tabular}{|lc|}
\hline lack of mouse tray & $35 \%$ \\
\hline lack of foot rest & $60 \%$ \\
\hline $\begin{array}{l}\text { Improper monitor } \\
\text { height }\end{array}$ & $80 \%$ \\
\hline
\end{tabular}

The other self reported ergonomics risk factors were lack of breaks (64\%) and stress (37\%). Based on statistical result we found that Myofascial Pain Syndrome $(49.20 \%)$ is the commonly affected musculoskeletal disorder among computer professionals. Other common diagnoses by physician were Fibromyalgia (8.5\%), Thoracic Outlet Syndrome (25.02\%), Cubital Tunnel Syndrome (0.16\%), Chronic Regional Pain Syndrome (0.20\%), and Wrist Tendinities $(3.70 \%)$. The percentage distributions were presented in Table 2.

The major prevalent body regions were neck $(64.9 \%)$, lower back $(56.5 \%)$, shoulder $(42.1 \%)$, thigh $(34.2 \%)$ followed by upper arm, forearm/wrist, knee and foot.

The result of the feedback regarding the SHARAN's protocol revealed that $95 \%$ of participants were well satisfied with the protocol and the treatment received in the on-site clinics. Only $5 \%$ of par- 
ticipants reported partial cure of their symptoms and still continuing with mild discomfort / pain.

\section{Discussion}

Studies have shown that neck, shoulders are the major body parts affected by MSD problems among the computer users [8]. The present study corroborates the result. Literature revealed that poor workstation can be major risk factors for the development of workrelated musculoskeletal disorders among the computer users [3]. The present study result also revealed similar kind of result where poor office ergonomics, and other furniture related issues were reported by the participants. In these onsite physical therapy clinics, $95 \%$ of patients achieved complete cure and 5\% partially cure. This result indicates that successfully treating employees with workrelated upper extremity disorders might be essential to achieving optimal success from the employer's perspective. We recommend an on-site clinic for the effective management of WRMSD. The employees who attended the clinics were on their acute or sub acute stages of pain or discomfort. The employees in their severe / chronic stage took more duration of intervention but cured completely. Also they can access the treatment facility in the early stages of condition with saving time. Major risk factors behind the WRMSD's are variations from workstations e.g. no key board tray, mouse on the writing surface etc. On site clinics allow immediate on-site workstation assessment and modifications, along with treatment will provide a permanent solution to the problem. The study finding suggests that onsite participants had fewer sickness absence hours; moreover, the economic evaluation suggests a difference in productivity loss at 6 and 12 months in favour of the onsite clinic participants. On-site employee health clinics are recommended for the effective prevention and management of WRMSD in view of the high prevalence of successful outcomes seen in this study.

\section{Conclusion}

We are concluding the advantages of on-site clinics include: 1. Convenience of employees, saving time and greater compliance, 2. Promoting earlier reporting of symptoms and hence a quicker and easier recovery, 3. Better follow up regarding recovery, work modifications and return to original work, 4. Allows on-site workstation assessment and modifications, 5. Maintenance of recovery by monitoring pos- ture, breaks and exercises, 6 . More effective coordination with members of Human Resources, Facilities, Health and Safety team, etc., 7. Improved awareness levels regarding Ergonomics among Management and Employees. Patients who did not achieve the goals had similar numbers of visits but a longer duration than patients who did achieve goals suggesting they may have more severe problems, poor compliance to follow treatment regimens or less access to the clinic due to their part-time work status.

\section{References}

[1] Bakhtiar C.S. and Vijaya R.S., Attitude Alters the Risk for Development of RSI in Software Professionals, Indian Journal. Occupational. Environmental Medicine, 2003, 7, 7 - 10.

[2] Bhanderi D., Choudhary S., Parmar L., and Doshi V., Influence of psychosocial workplace factors on occurrence of musculoskeletal discomfort in computer operators, Indian J Community Med, 2007, 32, 225-226.

[3] Marcus M., Gerr F., Monteilh C., Ortiz D.J., Gentry E., Cohen S., Edward A., Ensor C., Kleinbaum D., A prospective study of computer users: II Postural risk factors for musculoskeletal symptoms and disorders, American Journal of Industrial Medicine, 2002, 41, 236-249.

[4] Ming-Shun S.C., Benjamin C. A., Mary P. W., and Catherine D.R., Employer, Physical Therapist, and Employee Outcomes in the Management of Work-Related Upper Extremity Disorders, Journal of Occupational Rehabilitation, 2002, 12, 257 267

[5] Sharma A., Khera S., and Khandekar J., Computer related health problems among information technology professionals in Delhi, Indian J Community Med, 2006, 31, 36 - 38.

[6] Talwar R., Kapoor R., Puri K., Bansal K., and Singh S., A Study of Visual and Musculoskeletal Health Disorders among Computer Professionals in NCR Delhi, Indian J Community Med, 2009, 34, 326 - 328

[7] Wahlstro"m J., Ergonomics, musculoskeletal disorders and computer work, Occupational Medicine, 2005, 55, 168-176

[8] www.cyut.edu.tw.

[9] www.irsst.qc.ca 